\title{
How to obtain diagnostic and procedural quality three-dimensional-rotational angiograms in congenital heart disease: Tips and tricks from a single center experience
}

\author{
Jenny E. Zablah ${ }^{1,2}{ }^{\circ}$, Barry O’Callaghan ${ }^{1}$, Michael Shorofsky ${ }^{1}$, Gareth J. Morgan ${ }^{1,2}$ \\ ${ }^{1}$ Department of Pediatric Cardiology, Children's Hospital Colorado, Aurora, CO, United States \\ ${ }^{2}$ University of Colorado School of Medicine, Anschutz Medical Campus, Aurora, CO, United States
}

\section{The problem}

Three-dimensional rotational angiography (3DRA) is a commonly used imaging modality in congenital cardiac catheterization laboratories around the world. 3DRA research often focuses on the novelty and retrospective utility of 3DRA for a variety of procedures. Its hypothetical benefits may be through its potential to decrease radiation and contrast dose and to assist in the technical aspects of difficult cases [1-3]. There are currently no peer-reviewed guidelines on the efficient technical performance of a successful 3DRA, the key tenets of which are:

- Good quality angiography;

- Ease of 3D reconstruction;

- Minimizing catheter and foreign body artifact;

- Ability to use the resultant reconstructions to perform accurate measurements and produce a quantitatively representative image of structures;

- Accurate and rapid 3DRA reconstruction to facilitate overlay onto live fluoroscopy during procedural guidance.

\section{The proposal}

Extensive experience with current and past Philips's imaging technologies have allowed us to develop 3DRA protocols for different anatomical patient subsets, based on different structures and procedures of interest. These protocols, when used systematically provide high quality 3DRA using both the Philips' Allura and Azurion platforms. Sharing this experience may assist other centers in developing and improving their 3DRA workflows. The aim herein, was to provide guidance for other catheterization laboratories on useful tools and adjuncts that will allow them to take full advantage of the applications provided with their systems.

\section{Technical description}

Angiographic prescriptions for specific congenital lesions are summarized in Table 1. General recommendations for 3DRA include:

1. In our experience, using rapid ventricular pacing does not improve the quality of our diagnostic imaging, therefore we do not use it for 3DRA. Pacing alters the cardiac output, decreasing the accuracy of dimensional measurements. This can require the performance of additional $2 \mathrm{D}$ angiography to reliably measure structures of interest.

2. Lengthening the intravenous (IV) lines and ventilatory tubing/equipment to avoid any interaction with the $\mathrm{C}$-arm during the rotational angiogram. Excellent co-operation with the anesthesia team is essential.

3. 3DRA should be acquired during cessation of mechanical ventilation (expiratory breath hold) to eliminate respiratory motion artifacts.

Address for correspondence: Jenny E. Zablah, MD, Department of Pediatric Cardiology, Children's Hospital Colorado, 13123 E $16^{\text {th }}$ Ave, 80045 Aurora, CO 80045, United States, tel: 720-777-6140, e-mail: jenny.zablah@childrenscolorado.org 
Table 1. Children's Hospital Colorado three-dimensional rotational angiography (3DRA) protocols per cardiac lesion for Philips Allura and Azurion systems.

\begin{tabular}{|c|c|c|c|}
\hline & $<20 \mathbf{~ k g}$ & $20-50 \mathrm{~kg}$ & Adults (> 50 kg) \\
\hline \multicolumn{4}{|l|}{ Aortic arch } \\
\hline Catheter/size & Pigtail or Berman/4 F or $5 \mathrm{~F}$ & Pigtail or Berman $/ 5 \mathrm{~F}$ & Pigtail or Berman $/ 5 \mathrm{~F}$ or $6 \mathrm{~F}$ \\
\hline Injection site & Ascending aorta* & Ascending aorta* & Ascending aorta* \\
\hline Total volume & $2 \mathrm{~mL} / \mathrm{kg}$ & $2 \mathrm{~mL} / \mathrm{kg}$ & $100 \mathrm{~mL}$ \\
\hline Dilution ( $\%$ contrast) & $75 \%$ & $75 \%$ & $100 \%$ \\
\hline Delay time $[\mathbf{s}]$ & 1 & 1 & 1 \\
\hline Notes & \multicolumn{3}{|c|}{ Place catheter in LVOT instead of ascending aorta for improved coronary opacification. } \\
\hline \multicolumn{4}{|c|}{ I } \\
\hline \multirow[t]{2}{*}{ Catheter/size } & ${ }^{*}$ Long sheath $/ 5 \mathrm{~F}$ & ${ }^{*}$ Long sheath/6 F & ${ }^{*}$ Long sheath/6 F \\
\hline & Pigtail or Berman/4 F & Pigtail or Berman/4 F & Pigtail or Berman/4 F \\
\hline \multirow[t]{2}{*}{ Injection site } & IVC & IVC & IVC \\
\hline & SVC or innominate vein & SVC or innominate vein & SVC or innominate vein \\
\hline \multirow[t]{2}{*}{ Total volume } & $25 \%$ total volume by hand & $20 \mathrm{~mL}$ by hand & $20 \mathrm{~mL}$ by hand \\
\hline & $\begin{array}{c}1.5 \mathrm{~mL} / \mathrm{kg} \text { (minus the amount } \\
\text { given by hand) }\end{array}$ & $\begin{array}{c}1.5 \mathrm{~mL} / \mathrm{kg} \\
\text { (minus } 20 \mathrm{~mL} \text { ) }\end{array}$ & $\begin{array}{c}40 \mathrm{~mL} \\
\text { (for a total of } 60 \mathrm{~mL} \text { injection) }\end{array}$ \\
\hline Dilution ( $\%$ contrast) & $75 \%$ & $75 \%$ & $100 \%$ \\
\hline Delay time $[\mathbf{s}]$ & 1 & 1 & 1 \\
\hline Notes & \multicolumn{3}{|c|}{$\begin{array}{l}\text { Multiple site injection with IVC injections delineated in grey. Side arm injection by hand } \\
\text { into long sheath should be performed at same time as automated pump. We have also } \\
\text { used a short sheath for injection with good result. }\end{array}$} \\
\hline \multicolumn{4}{|c|}{ Superior cavopulmonary anastomosis (bidirectional glenn/hemifontan) } \\
\hline Catheter/size & Berman or Ssheath/5 F & Berman or sheath/5 F & \\
\hline Injection site & SVC or innominate vein & SVC or innominate vein & \\
\hline Total volume & $1 \mathrm{~mL} / \mathrm{kg}$ & $1 \mathrm{~mL} / \mathrm{kg}$ & \\
\hline Dilution (\% contrast) & $75 \%$ & $75 \%$ & \\
\hline Delay time $[\mathbf{s}]$ & 1 & 1 & \\
\hline Notes & \multicolumn{3}{|c|}{$1 \mathrm{~mL} / \mathrm{kg}$ is enough for an adequate 3DRA in a low flow system. } \\
\hline \multicolumn{4}{|c|}{ Pulmonary arteries (without stenosis and/or insufficiency) } \\
\hline Catheter/size & Berman or pigtail/5 F & Berman or pigtail/6 F & Berman or pigtail/6 F \\
\hline Injection site & RVOT & RVOT & RVOT \\
\hline Total volume & $2 \mathrm{~mL} / \mathrm{kg}$ & $2 \mathrm{~mL} / \mathrm{kg}$ & $100 \mathrm{~mL}$ \\
\hline Dilution (\% contrast) & $75 \%$ & $75 \%$ & $100 \%$ \\
\hline Delay time $[\mathrm{s}]$ & 1 & 1 & 1 \\
\hline \multicolumn{4}{|c|}{ Pulmonary arteries (with significant stenosis) } \\
\hline Catheter/size & Berman or pigtail/5 F & Berman or pigtail/6 F & Berman or pigtail/6 F \\
\hline Injection site & MPA & MPA & MPA \\
\hline Total volume & $2 \mathrm{~mL} / \mathrm{kg}$ & $2 \mathrm{~mL} / \mathrm{kg}$ & $100 \mathrm{~mL}$ \\
\hline Dilution (\% contrast) & $75 \%$ & $75 \%$ & $100 \%$ \\
\hline Delay time $[\mathbf{s}]$ & 1 & 1 & 1 \\
\hline Notes & \multicolumn{3}{|c|}{ Catheter side holes should be placed distal to vascular narrowing. } \\
\hline \multicolumn{4}{|c|}{ PPVI balloon coronary interrogation (non-selective) } \\
\hline Catheter/size & Pigtail/5 F & Pigtail/5 F & Pigtail/5 F or $6 \mathrm{~F}$ \\
\hline Injection site & Aortic root & Aortic root & Aortic root \\
\hline Total volume & $1.5 \mathrm{~mL} / \mathrm{kg}$ & $1.5 \mathrm{~mL} / \mathrm{kg}$ & $75 \mathrm{~mL}$ \\
\hline Dilution ( $\%$ contrast) & $75 \%$ & $75 \%$ & $100 \%$ \\
\hline Delay time $[\mathrm{s}]$ & 1 & 1 & 1 \\
\hline Notes & \multicolumn{3}{|c|}{$\begin{array}{l}\text { RVOT balloon contrast should be at } 50 \% \text { dilution. Injection to commence at full balloon } \\
\text { inflation. Lower injection volume in the setting of low cardiac output with RVOT balloon } \\
\text { occlusion. }\end{array}$} \\
\hline
\end{tabular}


Table 1 (cont.). Children's Hospital Colorado three-dimensional rotational angiography (3DRA) protocols per cardiac lesion for Philips Allura and Azurion systems.

\begin{tabular}{|c|c|c|c|}
\hline & $<20$ kg & $20-50$ kg & Adults (> 50 kg) \\
\hline \multicolumn{4}{|c|}{ PPVI balloon coronary interrogation (selective single coronary assessment) } \\
\hline Catheter/size & $\mathrm{JR}$ or JL/4 F & $\mathrm{JR}$ or JL/5 F & JR or JL/5 F \\
\hline Injection site & RCA or LCA & RCA or LCA & RCA or LCA \\
\hline Total volume & Manual injection & Manual injection & Manual injection \\
\hline Dilution ( $\%$ contrast) & $100 \%$ & $100 \%$ & $100 \%$ \\
\hline Delay time $[s]$ & 1 & 1 & 1 \\
\hline Notes & \multicolumn{3}{|c|}{$\begin{array}{l}\text { RVOT balloon contrast should be at } 50 \% \text { dilution. No pump angiography required. } \\
\text { Injection to commence at full balloon inflation with } 1 \mathrm{~s} \text { delay on } 3 \mathrm{D} \text { acquisition. } \\
\text { 'Coupling' should be switched off. }\end{array}$} \\
\hline \multicolumn{4}{|c|}{ PPVI (RVOT assessment in patients with stenosis or significant insufficiency) } \\
\hline Catheter / size & Pigtail or multitrack/5 F & Pigtail or multitrack/6 F & Pigtail or multitrack/6 F \\
\hline Injection site & MPA & MPA & MPA \\
\hline Total volume & $2 \mathrm{~mL} / \mathrm{kg}$ & $2 \mathrm{~mL} / \mathrm{kg}$ & $100 \mathrm{~mL}$ \\
\hline Dilution (\% contrast) & $75 \%$ & $75 \%$ & $100 \%$ \\
\hline Delay time $[s]$ & 1 & 1 & 1 \\
\hline Notes & \multicolumn{3}{|c|}{$\begin{array}{l}\text { If planning for overlay, perform the angiogram with your intended wire for the intervention } \\
\text { along with a Multitrack catheter. The stiff wire doesn't introduce significant artifact } \\
\text { and guidance is more accurate. }\end{array}$} \\
\hline
\end{tabular}

${ }^{*}$ Catheters are suggestions only; F — french; IVC — inferior vena cava; JL — left Judkins catheter; JR — right Judkins catheter; LCA — left coronary artery; LVOT — left ventricular outflow tract; MPA — main pulmonary artery; PPVI — percutaneous pulmonary valve implantation; RCA — right coronary artery; RVOT — right ventricular outflow tract; SVC — superior vena cava

4. When using 3DRA for fluoroscopic overlay during procedural guidance, we recommend having the procedural guide wire in its intended position for intervention. This will increase the accuracy of the overlay by cancelling out the anatomic shift which occurs after placing a stiff wire. Stationary guide wire does not produce a significant artifact.

5. For patients $<50 \mathrm{~kg}$, our contrast solution is diluted ( $75 \%$ contrast and $25 \%$ normal saline). Remember to agitate this solution prior to injection. For patients $>50 \mathrm{~kg}$, we use straight $(100 \%)$ contrast.

6. The catheter and accosted tubing should be primed with contrast prior to injection.

7. The contrast injection is given over $5 \mathrm{~s}$, starting $1 \mathrm{~s}$ before the $\mathrm{C}$-arm rotation begins $(1 \mathrm{~s}$ delay). This time delay should be modified when injecting in the pulmonary arterial circulation and focusing on levophase structures. The Philips platform has several available processing tools designed for vascular and solid organ imaging that we have found extremely useful in congenital cardiology (Fig. 1).

1. We perform $3 \mathrm{D}$ reconstructions using the XtraVision workstation (Philips Healthcare, Andover, Massachusetts, USA). During the re- construction, the first step before manipulating the histogram, is to remove any clips/wires or other highly opacified artifact.

2. We use XperCT to create multiplanar reconstructions of our imaging dataset facilitating measurements of structures of interest. These images are similar to multiplanar reconstructions views obtained with a computed tomographic angiography. This allows cardiac anatomy evaluation and its relationship with other nearby structures.

3. If the reconstruction will be used for live guidance overlay, we use the XperGuide function instead of the built-in "Overlay" tool. XperGuide was developed to guide solid organ procedures like liver or tumor biopsies. It allows the reconstructed 3DRA or a computed tomography "like" view (multiplanar view); to be overlaid on live fluoroscopy.

4. Using the "Segmentation" tool in the XtraVision workstation, we routinely segment airway anatomy, described in a prior publication [4]. This can be viewed along with the 3DRA vascular reconstruction and also overlaid onto live fluoroscopy using XperGuide.

In our experience, these "non-cardiac" software packages work well with acquisitions from 


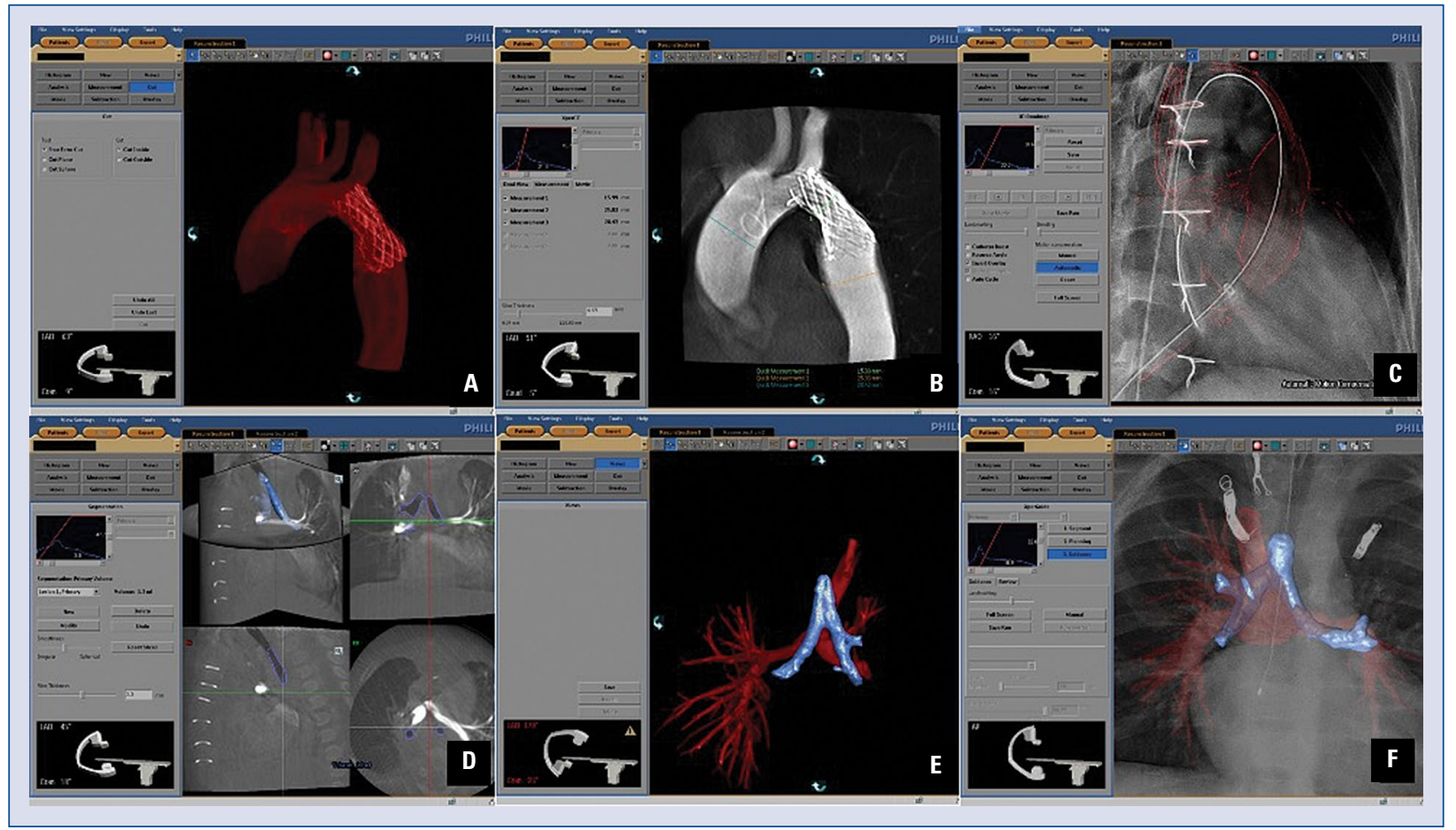

Figure 1. The multiple phases of three-dimensional rotational angiography (3DRA) acquisition, post processing, overlaying and segmentation; A. 3DRA reconstruction using Philips XtraVision workstation. The acquisition was in a patient with a coarctation of the aorta after stent placement. The angiogram was done with a Berman catheter to produce adequate contrast enhancement of entire aorta with minimal catheter related artifact; $\mathbf{B}$. XperCT is used to perform measurements in the multiplanar reconstruction (MPR) view of the 3DRA dataset, facilitating non-axial evaluation of the images; C. 3D Roadmap tool developed for the 3DRA overlay but unable to overlay airway and only allows the mask of the 3DRA to be used which limits the anatomic details available for live guidance (not used in our center, we use XperGuide instead); D. Segmentation tool that allows reconstruction of the airway with an MPR view; E. 3DRA reconstruction after the airway has been segmented. The airway can then be hidden to assess the vascular reconstruction only if necessary; F. XperGuide with 3DRA vascular and airway reconstruction overlay in live fluoroscopy.

both the Allura (FD10, FD20) and Azurion (B12/12) systems. Of note, using our current configurations, 3DRA acquisitions are performed at 15 frames per second with the Azurion system and 30 frames per second with the Allura system.

\section{Conclusions}

These simple guidelines on the proficient use of 3DRA with the Philips system have been developed over years of work with their angiographic platforms. They should act as guidance to clinicians who feel they are not utilizing their imaging systems fully as well as providing a technical platform for continued improvements generated by interested clinicians around the world.

Conflict of interest: None declared

\section{References}

1. Aldoss O, Fonseca BM, Truong UT, et al. Diagnostic utility of three-dimensional rotational angiography in congenital cardiac catheterization. Pediatr Cardiol. 2016; 37(7): 1211-1221, doi: 10.1007/s00246-016-1418-3, indexed in Pubmed: 27278632.

2. Zablah JE, Morgan GJ. Innovations in congenital interventional cardiology. Pediatr Clin North Am. 2020; 67(5): 973-993, doi: 10.1016/j.pcl.2020.06.012, indexed in Pubmed: 32888693.

3. Kang SL, Armstrong A, Krings G, et al. Three-dimensional rotational angiography in congenital heart disease: Present status and evolving future. Congenit Heart Dis. 2019; 14(6): 1046-1057, doi: 10.1111/chd.12838, indexed in Pubmed: 31483574 .

4. Góreczny S, Haak A, Morgan GJ, et al. Feasibility of airway segmentation from three-dimensional rotational angiography. Cardiol J. 2020; 27(6): 875-878, doi: 10.5603/CJ.a2020.0136, indexed in Pubmed: 33140395. 